\title{
Synergistic Anti-proliferative Effects of Metformin and Silibinin Combination on T47D Breast Cancer Cells via hTERT and Cyclin D1 Inhibition
}

\author{
Authors \\ Mina Chatran1, Younes Pilehvar-Soltanahmadi1, 2, 3, Mehdi Dadashpour1, 2, Leila Faramarzi1, 2, Sara Rasouli², \\ Davoud Jafari-Gharabaghlou ${ }^{4}$, Navid Asbaghi ${ }^{2}$, Nosratollah Zarghami 1, 2, 3, 4
}

\author{
Affiliations \\ 1 Department of Medical Biotechnology, Faculty of \\ Advanced Medical Sciences, Tabriz University of Medical \\ Sciences, Tabriz, Iran \\ 2 Hematology and Oncology Research Center, Tabriz \\ University of Medical Sciences, Tabriz, Iran \\ 3 Stem Cell Research Center, Tabriz University of Medical \\ Sciences, Tabriz, Iran \\ 4 Department of Clinical Biochemistry and Laboratory \\ Sciences, Faculty of Medicine, Tabriz University of \\ Medical Sciences, Tabriz, Iran
}

Key words

Breast cancer, Synergistic effect, Metformin, Silibinin, hTERT

received 31.08 .2017

accepted 15.05.2018

\author{
Bibliography \\ DOI https://doi.org/10.1055/a-0631-8046 \\ Published online: 19.6.2018 \\ Drug Res 2018; 68: 710-716 \\ (c) Georg Thieme Verlag KG Stuttgart · New York \\ ISSN 2194-9379
}

\author{
Correspondence \\ Nosratollah Zarghami, PhD \\ Department of Medical Biotechnology \\ Faculty of Advanced Medical Sciences \\ Tabriz University of Medical Sciences \\ Golgasht Street \\ 51656-65811 Tabriz \\ Iran \\ Tel.: + 98/413/33355 788, Fax: + 98/413/33355 788 \\ zarghami@tbzmed.ac.ir
}

\section{ABSTRACT}

Background There is a growing body of data that chemotherapeutic combination strategies would be more effective in reducing drug toxicity, inhibiting tumor progression in comparison to either drug alone.

Objective To explore a chemopreventive strategy for improving breast cancer treatment efficacy, the anticancer effects of a combination of Metformin (MET) and Silibinin (SIL) were investigated in T47D breast cancer cells.

Materials and Methods Cytotoxicity of the drugs individually and in combination was evaluated using MTT assay. The precise nature of the interaction between MET and SIL was further analyzed through the median-effect method. In addition, qRT-PCR was applied to determine the expression levels of hTERT and cyclin D1 genes after $48 \mathrm{~h}$ drug exposure.

Results MTT assays showed that MET and SIL individually inhibited the cell viability in a dose and time-dependent manner, and the obtained combination indices ( $\mathrm{Cls}$ ) were $<1$ for all the combination treatments, indicating that the anticancer agents synergistically induced growth inhibition in the breast cancer cells. QPCR findings revealed that the drug combination also synergistically down-regulated the expression levels of hTERT and cyclin D1 at all used concentrations compared with the drugs used alone after $48 \mathrm{~h}$ treatment $(\mathrm{P} \leq 0.05)$.

Conclusion The results provide evidence that synergistic antiproliferative effects of MET and SIL, linking to the down-regulation of Cyclin D1 and hTERT genes, and propose that $\mathrm{MET}+\mathrm{SIL}$ may have therapeutic value in breast cancer therapy.

\section{Introduction}

About $12 \%$ U.S. women will develop invasive breast cancer over the during her lifetime. In 2017, an estimated 252,710 new cases of invasive breast cancer are predictable to be identified in U.S. women, along with 63,410 new cases of non-invasive breast cancer [1].
Breast cancer is considered as a heterogeneous disease with several clinical features and risk factors. Breast lumps, shape change in breast, skin dimpling, nipple discharge, a scaly or red skin patch, swollen lymph nodes, bone pain and breath shortness are the common signs of breast cancer [2]. Established risk factors for develop- 
ing breast cancer include variable life style factors including being female, obesity, lack of physical exercise, hormone replacement therapy and alcohol consumption, and; reproductive factors such as low parity and breast-feeding, late menopause and early menarche,; and inherent genetic factors [3].

Although several chemotherapeutics such as doxorubicin, etoposide, and paclitaxel have been used to treat this type of cancer, issues remain such as low survival rates, high reoccurrence and severe side effects after conventional chemotherapy. Thus, novel chemotherapeutics and approaches should be developed [4,5].

Nowadays, numerous natural bioactive compounds or phytochemicals have been isolated, described, and their potential anticancer effects have been evaluated [6-9]. Although, efficiency of interactions between various dietary compounds needs further investigation.

Silibinin (SIL), a flavonolignan extracted from Silybum marianum, has been widely investigated for its hepatoprotective, antiinflammatory, antioxidant and anticancer properties (structures shown in > Fig. 1a) $[10,11]$. Furthermore, it has demonstrated considerable efficiency in inhibiting or delaying both tumor initiationand promotion-related incidences in various pre-clinical cancer models such as prostate, colorectal, lung and breast cancer $[12,13]$. Also, recent studies show that combination of SIL with some substances can enhance the therapeutic properties $[4,14]$.

Also, several recent preclinical and clinical data all suggest that metformin (MET) as a biguanide originated from the French lilac or goat's rue (Galega officinalis) (structures shown in > Fig. 1b) with known pharmacokinetics, high safety profiles, and relatively inexpensive might be effective against various types of cancer including breast cancer $[5,15,16]$.

More recent cancer therapies are established on an effective combination of various chemotherapeutics with different mechanisms. A combination approach may increase the anti-cancer effects or even results in a synergic inhibitory effect on a causal and contributing factor in carcinogenesis. This strategy, specifically in the removal of hindrances such as low drug efficiency and tumor resistance, can be valuable to improve the total therapeutic effectiveness of the single drug $[17,18]$. Currently, CompuSyn has become a popular tool to calculate a combination index $(\mathrm{Cl})$ through Chou-Talalay method. This method for drug combination is based on the median-effect equation, derived from the mass-action law principle, which is the unified theory that provides the link between single entity and multiple entities, and first order and higher order dynamics. This common equation includes the Michaelis-Menten, Scatchard, Henderson-Hasselbalch and Hill equations in biophysics and biochemistry [19].

Cyclin D1 overexpression is found in more than $50 \%$ of human breast cancers and leads to mammary cancer in transgenic mice. In addition to inducing progression of cell cycle via cyclin-dependent kinases (CDKs) activity regulation, cyclin D1 promotes other regulatory molecules by CDK-independent mechanisms [19]. Transcriptional activity of signal transducer and activator of transcription 3 (STAT3) was suppressed by cyclin D1. Losing antiapoptotic activity of STAT3 and causing apoptosis induction. Cyclin D1 plays role in as a intermediary metabolite in other cell cycle control pathways including, Rac1, 5' adenosine monophosphate- activated protein kinase (AMPK) and nuclear factor- B (NFKB) signaling pathways. reduction in Rac1 levels causes NFKB signaling inhibition and prompts decrease of cyclin D1. Active AMPK results in loss of cyclin D1 in mRNA and protein levels [20].

Telomerase has been known as an attractive therapeutic target for treatment of different cancers, as it preserves tumor cells division and survival and decreases apoptosis induction. It has been shown that telomerase is active in $90 \%$ of breast carcinomas and $85 \%$ of human cancers, while in normal cells it is not active or detectable [21, 22]. It was reported that telomerase reactivation was considerably related with advanced breast cancer stage, nodal metastasis and histopathological grade, and no significant association between telomerase activity and menopausal status, or tumor size was found. Besides, it was revealed that no significant association between tumor hTERT (human telomerase reverse transcriptase) expression and patient's age, tumor size, grade, nodal metastasis, estrogen receptor (ER) positivity and lymphovascular (LVI) [23, 24]. Thus, Inhibition of telomerase activity specially its catalytic subunit, hTERT, in breast cancer cells may reactivate telomere shortening and might be a hopeful target in breast cancer treatment $[15,25]$.

Regarding to the demonstrated anti-cancer effects of MET and SIL as safe anticancer agents and lack of studies showing combined inhibitory effects of the drugs, The aim of this study was to evaluate the hypothesis that Silibinin and Metformin will work in synergism and inhibit the growth of T47D cells as a model breast cancer cell line. Thus, Silibinin, Metformin and their combination in constant ratios with regard to their IC50s were used to determine the cytotoxicity and expression levels of hTERT and Cyclin D1 in T47D breast cancer cells.

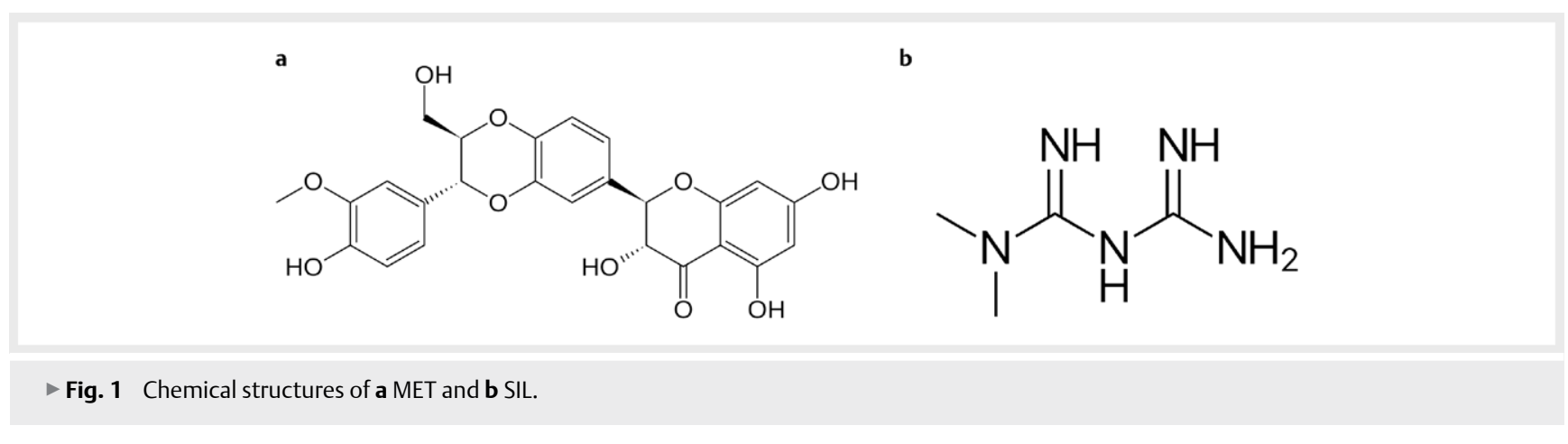




\section{Materials and Methods}

\section{Chemicals and reagents}

Silibinin, Metformin and MTT powder were obtained from Sigma (Deisenhofen, Germany); T47D breast cancer cell line purchased from the Pasteur Institute of Iran, Tehran, Iran; fetal bovine serum (FBS) and phenol-red free RPMI 1640 with $L$ glutamine were purchased from Gibco BRL (Life Technologies, Grand Island, NY, USA); Sodium bicarbonate and Streptomycin were purchased from Merck (Darmstadt, Germany); Penicillin G was purchased from SERVA (Heidelberg, Germany); TRIZOL reagent was purchased from Invitrogen (Eugene, OR, USA); First-Strand cDNA Synthesis kit was purchased from Fermentas (Hanover, MD, USA); and Syber Green-I reagent was purchased from Takara Bio (Otsu, Japan).

\section{In vitro cytotoxicity assay}

RPMI 1640 medium supplemented with $10 \%$ FBS and $1 \%$ penicillin/streptomycin were used to culture T47D breast cancer cells. The cells were incubated in a humidified atmosphere containing $5 \%$ $\mathrm{CO}_{2}$ at $37^{\circ} \mathrm{C}$.

Cytotoxic activity was studied using MTT cell viability assay after 24 and 48 h treatment of MET, SIL and MET + SIL to the cells. Metabolically active cells decrease the tetrazolium constituent of MTT in to purple colored formazan crystals. Briefly, $2 \times 10^{4}$ cells/well were cultivated in 96 -well plates for $24 \mathrm{~h}$ and then, treated with different concentrations of MET $(0,1,5,10,15,20$ and $25 \mathrm{mM})$, SIL $(0,5,25,50,75,100$ and $125 \mu \mathrm{M})$ and MET + SIL (1000:5, 5000:25,

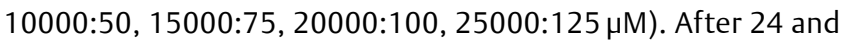
$48 \mathrm{~h}$ exposure time, medium was replaced with fresh medium and $2 \mathrm{mg} / \mathrm{ml}$ of MTT was added to each well and plates were covered with aluminum foil and incubated for $4 \mathrm{~h}$ at $37^{\circ} \mathrm{C}$. Thereafter, the content of the wells was removed and $200 \mu \mathrm{L}$ pure DMSO and $25 \mu \mathrm{L}$ Sorensen's glycine buffer were added. In the next step, absorbance of each well was read at $570 \mathrm{~nm}$ using ELISA plate reader (Bio-Tek Instruments) with reference wavelength of $630 \mathrm{~nm}$ [26].

IC50s of MET, SIL and MET + SIL against T47D were measured using GraphPad Prism version 6.7 (GraphPad Software, Inc., San Diego, CA, USA). Also, Compusyn software version 1.0 (ComboSyn, Inc.) were used to determine the nature of interaction between MET and SIL in combination form

\section{RNA extraction, CDNA synthesis and Real-time PCR}

qRT-PCR assay was used to analyze expression levels of relative mRNA of hTERT and Cyclin D1 genes. T47D cells were treated with different concentrations of MET, SIL and MET + SIL for $48 \mathrm{~h}$. After drug exposure time, total RNA was isolated using the Trizol reagent according manufactures protocol. Then, the quantity and quality of total RNA was assessed based on $\mathrm{OD}_{260 / 280}$ ratio measurements and electrophoresis on $1.5 \%$ agarose, respectively.

Equal amount of RNA was taken from all the samples and reverse transcribed using RevertAid First strand cDNA synthesis Kit (Fermentas, St Leon-Rot, Germany) to gain complementary DNA (cDNA). The reverse transcription reaction product can be directly used in PCR applications or stored at $-20^{\circ} \mathrm{C}$ for less than one week. For longer storage, $-70^{\circ} \mathrm{C}$ is recommended.

hTERT and cyclin D1 gene expression in T47D breast cancer cells after exposure to MET, SIL and MET + SIL for $48 \mathrm{~h}$ was assessed by real-time PCR. The synthesized cDNA was diluted 1:5, 1:10 and $1: 20$. The concentration of $1: 20$ of cDNA was used as template for real-time PCR. Then, the synthesized cDNA was amplified by quantitative real-time RT-PCR using specific primers (Takapou Zist Co., Iran) and the SYBR Green-I dye (Roche, Germany) by the Rotor-GeneTM 6000 system (Corbett research, Australia). The quality of real-time PCR reactions was controlled by running standard samples as duplicated 5 times serial dilutions of cDNA obtained from the T47D breast cell line served as samples with strong expression of hTERT gene. The program for real-time PCR reaction was as follows; Initial denaturation at $95^{\circ} \mathrm{C}$ for $10 \mathrm{~min}$, followed by cycles of denaturation at $95^{\circ} \mathrm{C}$ for $15 \mathrm{~s}$, annealing at $60^{\circ} \mathrm{C}$ for $30 \mathrm{~s}$ and extension at $72^{\circ} \mathrm{C}$ for $30 \mathrm{~s}$. Finally, amplicons were measured by melting curve analysis of $70^{\circ} \mathrm{C}$ to $95^{\circ} \mathrm{C}$. The real-time PCR efficiencies were determined for each gene. Relative hTERT and cyclin D1 expression levels was normalized by housekeeping gene ( $\beta$-actin) and relative expression of the genes calculated by this formula: (normalized relative ratio $=2-\mathrm{Ct}$ ).

The program for real-time PCR reaction was as follows; Initial denaturation at $95^{\circ} \mathrm{C}$ for $15 \mathrm{~min}$ ( 1 cycle), followed by cycles of denaturation at $95^{\circ} \mathrm{C}$ for $15 \mathrm{~s}$ ( 45 cycle), annealing at $59^{\circ} \mathrm{C}$ for $30 \mathrm{~s}$ and extension at $72^{\circ} \mathrm{C}$ for $20 \mathrm{~s}$. Finally, amplicons were assessed by melting curve analysis of $59-95^{\circ} \mathrm{C}$ to for $5 \mathrm{~s}$ for each degree ( 1 cycle).

\section{Statistical analysis}

All experiments were done in three replicates and values displayed are representative for at least three independent experiments. Graph Pad Prism 6.7 was used for statistical analysis. All the results of the experiments were expressed as the mean \pm standard deviation (Mean $\pm \mathrm{SD}$ ). Levels of the statistical significance were measured using the paired Student $t$ test when comparing two groups, or by analysis of variance (ANOVA). $P$ values of $P \leq 0.05$ were considered significant.

\section{Results}

\section{In vitro cytotoxicity}

The effect of free MET, free SIL and MET + SIL on growth inhibition of T47D were examined using colorimetric MTT assay after 24 and $48 \mathrm{~h}$ treatment. the cytotoxicity assay findings revealed that both MET and SIL significantly inhibited the proliferation of T47D cells growth in a time and dose-dependent manner ( $>$ Fig. 2 ).

- Table 1 displays the half maximal inhibitory concentration $\left(\mathrm{IC}_{50}\right)$ and combination index $\left(\mathrm{Cl}_{50}\right)$ values for the drug formulations against T47D and for 24 and $48 \mathrm{~h}$ incubation time. According to the data analysis of the cytotoxicity assay, the combination of MET and SIL showed more growth inhibitory effect with a drastic reduction in IC50s of MET and SIL in the breast cancer cells than the single treatments after 24 and 48 incubation time.

CompuSyn software was used to analysis the precise nature of the interaction between MET and SIL in combination through Median-effect method. Based on this method, the obtained combination index plot showed that the $\mathrm{Cl}_{50}$ values of MET + SIL for T47D breast cancer cells were measured to be 0.86 and 0.63 , respective- 
a

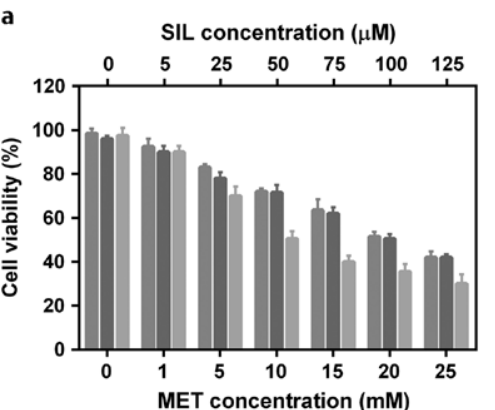

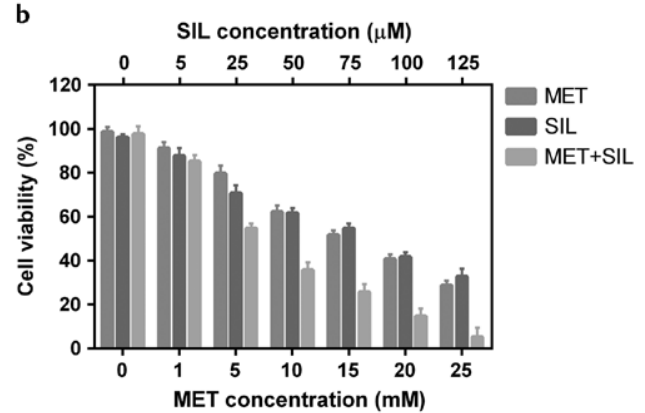

Fig. 2 Assessment of antiproliferative effects of MET, SIL and MET + SIL on T47D breast cancer cells using MTT assay after $\mathbf{a} 24$ and $\mathbf{b} 48 \mathrm{~h}$ treatment. Data shown are representative of three independent experiments.

- Table $1 \quad \mathrm{IC}_{50}$ and combination index $\left(\mathrm{Cl}_{50}\right)$ values for the drug formulations against T47D and MDA-MB-231 cells for $48 \mathrm{~h}$ incubation time.

\begin{tabular}{|l|c|c|c|c|c|}
\hline $\begin{array}{l}\text { Time } \\
(\mathbf{h})\end{array}$ & $\begin{array}{c}\mathrm{IC}_{50} \\
\mathrm{MET} \\
(\mathrm{mM})\end{array}$ & $\begin{array}{c}\mathrm{IC}_{50} \mathrm{SIL} \\
(\boldsymbol{\mu} \mathrm{M})\end{array}$ & $\begin{array}{c}\mathrm{IC}_{50} \text { of } \\
\text { MET in } \\
\text { combi- } \\
\text { nation } \\
(\mathbf{m M})\end{array}$ & $\begin{array}{c}\mathrm{IC}_{50} \text { of } \\
\text { SIL in } \\
\text { com- } \\
\text { bina- } \\
\text { tion } \\
(\boldsymbol{\mu} \mathrm{M})\end{array}$ & $\mathrm{Cl}_{50}$ \\
\hline 24 & 21.20 & 106.50 & 10.60 & 53.01 & 0.862 \\
\hline 48 & 14.34 & 72.04 & 5.543 & 27.72 & 0.631 \\
\hline
\end{tabular}

ly, indicating the synergistic growth inhibitory effect of MET and SIL in combination form in a time dependent manner ( $\triangleright$ Fig. 3 ).

\section{Gene expression findings}

To further explore the mechanisms involved in MET and SIL combination-mediated inhibition on T47D breast cancer cells, qRT-PCR was applied to measure the expression levels of hTERT and cyclin D1 genes. Therefore, the expression levels of the genes was determined after $48 \mathrm{~h}$ drug treatment of breast cancer cells. Real-time $P C R$ results showed that exposure of T47D cells to the various concentrations of MET and SIL lead to a significant reduction in the expression levels of hTERT and cyclin D1 as a dose dependent manner in compared with control group. As shown in > Fig. 4, Importantly, hTERT and cyclin D1 mRNA levels decreased highly in the combined treatment groups in all applied concentration in relative to the groups for which drugs were applied alone $(P \leq 0.05)$.

\section{Discussion}

In the current work, the cytotoxicity of MET + SIL were assessed against T47D breast cancer cells. The assessment disclosed high capability of the combination to diminish the growth of T47D breast cancer cells through inhibition of hTERT and cyclin D1 expression levels.

It was found that single treatments of MET and SIL exhibited proliferative inhibitory effect against breast cancer cells in a dose- and time-dependent manner. The results are in harmony with those from previous studies which displayed the antiproliferative activity of MET + SIL against different types of breast cancer cell lines.
In this study, it was evaluated whether combining MET with SIL has a greater cytotoxicity against T47D breast cancer cells compared to the single treatments of the anticancer agents. The results propose that MET + SIL were strongly effectual in killing the breast cancer cells.

The quiddity of the combination effect was further analyzed by the median-effect method [27]. A series of "dose (D) and effect ( $\mathrm{fa}$ )" were entered into CompuSyn for each drug alone and their combination, the software automatically simulated the $\mathrm{Cl}$ values at different fa levels in seconds, based on the $\mathrm{Cl}$ algorithm. The resulting $\mathrm{Cl}$ theorem of Chou-Talalay offers quantitative definition for additive effect $(\mathrm{Cl}=1)$, synergism $(\mathrm{Cl}<1)$, and antagonism $(\mathrm{Cl}>1)$ in drug combinations. According to our results, The $\mathrm{Cls}$ value were $<1$ for the combination treatments after 24 and $48 \mathrm{~h}$, indicating an inhibitory synergistic effect of MET + SIL against proliferation of T47D breast cancer cells.

The effect of SIL in combination with various anti-cancer agents such as cisplatin, doxorubicin, carboplatin, and curcumin against breast cancer cells has been studied. Combination of SIL with doxorubicin caused a stronger apoptotic death in MCF-7 and MDAMB468 breast cancer cells compared to either drug alone. Besides, SIL in combination with carboplatin resulted in a much stronger apoptotic effect in MCF-7 cells. On the other hand, SIL plus cisplatin revealed no further apoptotic activity in MCF-7 and MDAMB468 cells [28]. Combination of SIL with curcumin also inhibited the proliferation and telomerase expression of T47D breast cancer cells [12]. Hence, It was suggested that SIL + curcumin might be a hopeful therapeutic approach in breast cancer therapy.

Also, the combination of MET with chemotherapeutic agents such as paclitaxel, carboplatin, epirubicin, doxorubicin, 5-FU, and cyclophosphamide have been extensively reported [29-31]. Recent data showed that MET in combination with trastuzumab killed cancer stem cells and inactivated ErbB2/IGF-1 R interactions in a synergistic manner via inhibiting Src kinase and/or PI3K/Akt pathway, causing overwhelming primary resistance to trastuzumab in HER2 positive breast cancer cells [32,33]. Ma and colleagues showed that MET reduced migration and invasion of cancer cells in tamoxifen-resistant breast cancer cells and in combination with tamoxifen synergistically inhibited proliferation of ER positive breast cancer via the $\mathrm{Bax} / \mathrm{Bcl}-2$ and $\mathrm{AMPK} / \mathrm{mTOR} / \mathrm{p} 70 \mathrm{S6K}$ pathways $[34,35]$. It was found that the combination treatment of MET and 
a

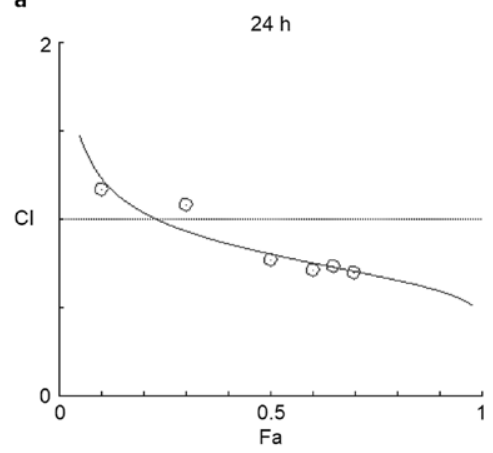

b

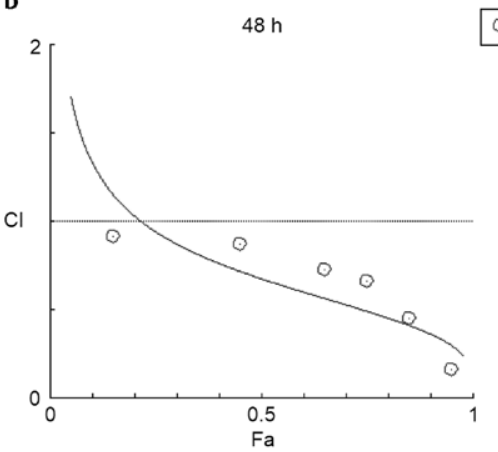

- Fig. 3 Synergistic growth inhibitory effects of MET + SIL on T47D breast cancer cells. Combination index (CI) was calculated by isobologram analysis using the Chou-Talalay method. $\mathrm{Cl}=1$, additive effect; $\mathrm{Cl}<1$, synergistic effect; $\mathrm{Cl}>1$, antagonistic effect. Data represented are from three independent experiments.

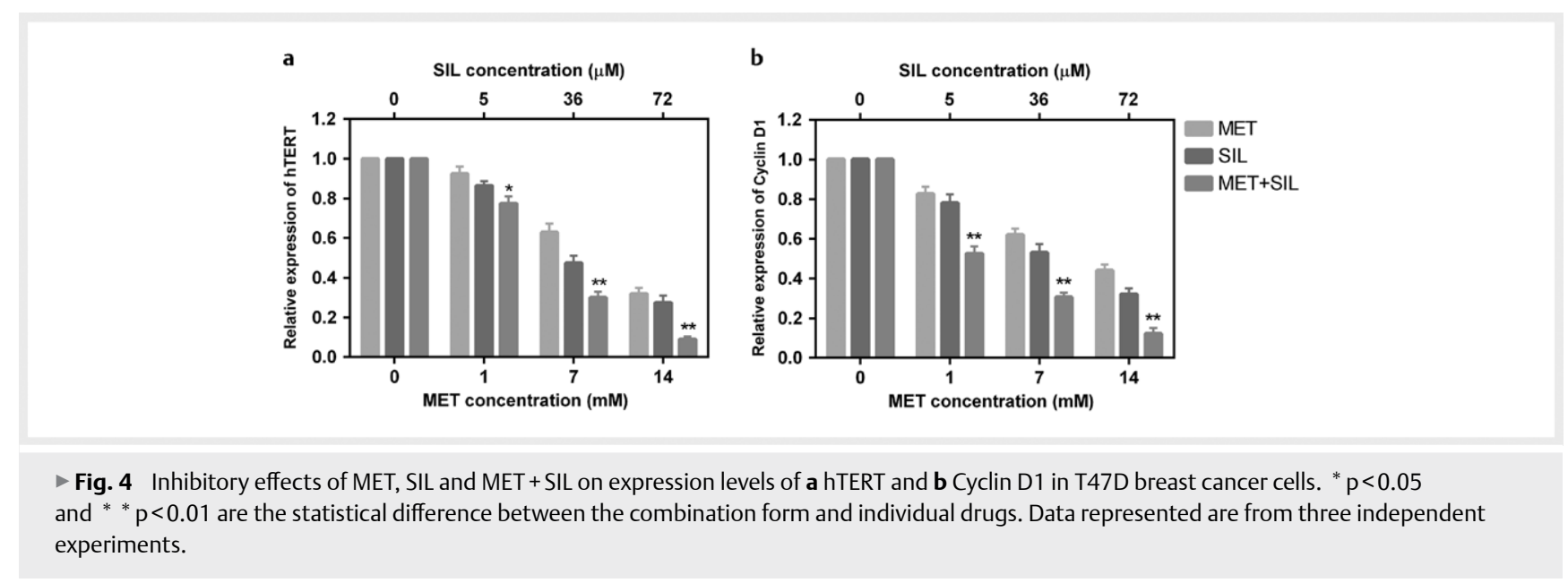

paclitaxel arrested in $\mathrm{G} 2 / \mathrm{M}$ phase, increased apoptosis and reduced cell proliferation in MCF-7 breast cancer cells [36].

To explore the mechanisms involved in MET and SIL combination-mediated inhibition on breast cancer cells, the expression levels of hTERT and cyclin D1 genes were assessed and our data suggested that the synergistic cytotoxic activity of the combination involves simultaneous inhibition of hTERT and cyclin D1 expression.

Based on a study by Cantrell et al., telomerase activity can be regulated through MET treatment in various cancer cells. It was shown that MET strongly inhibit proliferation of endometrial cancer cells in a dose dependent manner. Moreover, MET caused hTERT reduction, G1 arrest and apoptosis induction [37].

Among the different cancer models, the effects of silibinin have been strongly evaluated in the prevention and growth control of breast cancer through extensive in vitro and in vivo. studies conducted in our laboratory and other researchers. Based on our previous studies, it has been revealed that SIL inhibited proliferation of T47D breast cancer cells through reduction of TERT expression as well as telomerase activity $[11,12]$. Also, SIL has been shown to potently inhibit breast cancer through targeting multiple cell signaling pathways, inducing apoptosis, and inhibiting invasion, metastasis, and angiogenesis [38, 39].
In this study, we surveyed the alteration in expression levels of cyclin D1 and hTERT genes to describe the synergistic anticancer effects of MET and SIL in molecular level. However, further studies are needed to provide insight into the mechanisms involved in the elicited anticancer effects of the combination treatment of MET and SIL. Also, studies showed that unfavorable properties of these drugs such as poor solubility in water and low cellular uptake can limit the effectiveness of the drugs. So, using co-nanodelivery systems may enhance bioavailability of these natural therapeutic agents and result in strong synergistic anticancer effects on breast cancer cells.

\section{Conclusion}

In conclusion, the current study showed that the combination of MET and SIL exerts synergistically growth inhibitory effects against T47D breast cancer cells through down-regulation of hTERT and Cyclin D1 expression levels. Based on this finding, it can be suggested that the combinatorial chemotherapy based on MET and SIL may be a rationale and effective strategy for breast cancer therapy. 


\section{Acknowledgments}

The authors would like to thank Department of Medical Biotechnology, Faculty of Advanced Medical Sciences, Tabriz University of Medical Sciences for supporting this project.

\section{Conflict of Interest}

The authors declare that they have no conflict of interest.

\section{References}

[1] DeSantis CE, Fedewa SA, Goding Sauer A et al. Breast cancer statistics, 2015: Convergence of incidence rates between black and white women. CA: A Cancer Journal for Clinicians 2016; 66: 31-42

[2] Runowicz CD, Leach CR, Henry NL et al. American cancer society/ American society of clinical oncology breast cancer survivorship care guideline. CA: A Cancer Journal for Clinicians 2016; 66: 43-73

[3] Vos S, Van der Groep P, Van der Wall E et al. Hereditary Breast Cancer Syndromes: Molecular Pathogenesis and Diagnostics. eLS 2015

[4] Maasomi Z], Pilehvar-Soltanahmadi Y, Dadashpour M et al. Synergistic anticancer effects of silibinin and chrysin in T47D breast cancer cells. Asian Pacific Journal of Cancer Prevention: APJCP 2017; 18: 1283

[5] Farajzadeh R, Pilehvar-Soltanahmadi Y, Dadashpour M et al. Nanoencapsulated metformin-curcumin in PLGA/PEG inhibits synergistically growth and hTERT gene expression in human breast cancer cells. Artificial Cells, Nanomedicine, and Biotechnology 2017; 1-9

[6] Alibakhshi A, Ranjbari J, Pilehvar-Soltanahmadi Y et al. An update on phytochemicals in molecular target therapy of cancer: Potential inhibitory effect on telomerase activity. Current Medicinal Chemistry 2016; 23: 2380-2393

[7] Mohammadian F, Pilehvar-Soltanahmadi Y, Zarghami F et al. Upregulation of miR-9 and Let-7a by nanoencapsulated chrysin in gastric cancer cells. Artificial Cells, Nanomedicine, and Biotechnology 2017; 45: 1201-1206

[8] Montazeri M, Pilehvar-Soltanahmadi Y, Mohaghegh M et al. Antiproliferative and apoptotic effect of dendrosomal curcumin nanoformulation in P53 mutant and wide-type cancer cell lines. Anti-Cancer Agents in Medicinal Chemistry (Formerly Current Medicinal Chemistry-AntiCancer Agents) 2017; 17: 662-673

[9] Dadashpour M, Pilehvar-Soltanahmadi Y, Zarghami N et al. Emerging importance of phytochemicals in regulation of stem cells fate via signaling pathways. Phytotherapy Research 2017

[10] Ebrahimnezhad Z, Zarghami N, Keyhani M et al. Inhibition of hTERT gene expression by silibinin-loaded PLGA-PEG-Fe3O4 in T47D breast cancer cell line. Biolmpacts: BI 2013; 3: 67

[11] Amirsaadat S, Pilehvar-Soltanahmadi Y, Zarghami F et al. Silibininloaded magnetic nanoparticles inhibit hTERT gene expression and proliferation of lung cancer cells. Artificial Cells, Nanomedicine, and Biotechnology 2017; 45: 1649-1656

[12] Nasiri M, Zarghami N, Koshki KN et al. Curcumin and silibinin inhibit telomerase expression in T47D human breast cancer cells. Asian Pacific Journal of Cancer Prevention 2013; 14: 3449-3453

[13] Yurtcu E, Iseri OD, Sahin FI. Effects of silymarin and silymarindoxorubicin applications on telomerase activity of human hepatocellular carcinoma cell line HepG2. J BUON 2015; 20: 555-561

[14] Sun H-p, Su J-H, Meng Q-S et al. Silibinin and indocyanine green-loaded nanoparticles inhibit the growth and metastasis of mammalian breast cancer cells in vitro. Acta Pharmacologica Sinica 2016; 37: 941
[15] Lotfi-Attari ], Pilehvar-Soltanahmadi Y, Dadashpour M et al. Co-Delivery of Curcumin and Chrysin by Polymeric Nanoparticles Inhibit Synergistically Growth and hTERT Gene Expression in Human Colorectal Cancer Cells. Nutrition and Cancer 2017; 69: 1290-1299

[16] Javidfar S, Pilehvar-Soltanahmadi Y, Farajzadeh R. et al. The inhibitory effects of nano-encapsulated metformin on growth and hTERT expression in breast cancer cells. Journal of Drug Delivery Science and Technology 2017

[17] Pinto AC, Moreira JN, Simões S. Combination chemotherapy in cancer: Principles, evaluation and drug delivery strategies, in Current Cancer Treatment-Novel Beyond Conventional Approaches. 2011, InTech

[18] Tavakoli F, Jahanban-Esfahlan R, Seidi K et al. Effects of nano-encapsulated curcumin-chrysin on telomerase, MMPs and TIMPs gene expression in mouse B16F10 melanoma tumour model. Artificial Cells, Nanomedicine, and Biotechnology 2018; 1-12

[19] Chou T-C. Drug combination studies and their synergy quantification using the Chou-Talalay method. Cancer Research 2010; 70: 440-446

[20] Burandt E, Grünert M, Lebeau A et al. Cyclin D1 gene amplification is highly homogeneous in breast cancer. Breast Cancer 2016; 23 : $111-119$

[21] Sadeghzadeh H, Pilehvar-Soltanahmad Y, Akbarzadeh A et al. The effects of nanoencapsulated curcumin-Fe3O4 on proliferation and hTERT gene expression in lung cancer cells. Anti-Cancer Agents in Medicinal Chemistry (Formerly Current Medicinal Chemistry-AntiCancer Agents) 2017; 17: 1363-1373

[22] Pirmoradi S, Fathi E, Farahzadi R et al. Curcumin affects adipose tissue-derived mesenchymal stem cell aging through TERT gene expression. Drug Research 2018; 68: 213-221

[23] Kirkpatrick K, Ogunkolade W, Elkak A et al. hTERT expression in human breast cancer and non-cancerous breast tissue: correlation with tumour stage and c-Myc expression. Breast Cancer Research and Treatment 2003; 77: 277-284

[24] Mokbel K, Parris CN, Ghilchik M et al. The association between telomerase, histopathological parameters, and $\mathrm{KI}-67$ expression in breast cancer. The American Journal of Surgery 1999; 178: 69-72

[25] Wang L, Soria J-C, Kemp BL et al. hTERT expression is a prognostic factor of survival in patients with stage I non-small cell lung cancer. Clinical Cancer Research 2002; 8: 2883-2889

[26] Mohammadian F, Abhari A, Dariushnejad $\mathrm{H}$ et al. Upregulation of Mir-34a in AGS gastric cancer cells by a PLGA-PEG-PLGA chrysin nano formulation. Asian Pac J Cancer Prev 2015; 16: 8259-8263

[27] Fu J, Chou T-C. Abstract 4554 A: Simple, efficient, and quantitative approach for determination of synergism, additive effect, and antagonism of drugs in vivo using combination index method: A proposition for clinical protocol design and regulatory synergy claims. 2017, AACR

[28] Tyagi AK, Agarwal C, Chan DC et al. Synergistic anti-cancer effects of silibinin with conventional cytotoxic agents doxorubicin, cisplatin and carboplatin against human breast carcinoma MCF-7 and MDA-MB468 cells. Oncology Reports 2004; 11: 493-499

[29] Iliopoulos D, Hirsch HA, Struhl K. Metformin decreases the dose of chemotherapy for prolonging tumor remission in mouse xenografts involving multiple cancer cell types. Cancer Research 2011; 71: 3196-3201

[30] Liu H, Scholz C, Zang C et al. Metformin and the mTOR inhibitor everolimus (RAD001) sensitize breast cancer cells to the cytotoxic effect of chemotherapeutic drugs in vitro. Anticancer Research 2012; 32: $1627-1637$

[31] Soo JS-S, Ng C-H, Tan SH et al. Metformin synergizes 5-fluorouracil, epirubicin, and cyclophosphamide (FEC) combination therapy through impairing intracellular ATP production and DNA repair in breast cancer stem cells. Apoptosis 2015; 20: 1373-1387 
[32] Vazquez-Martin A, Oliveras-Ferraros C, Del Barco S et al. et al. The anti-diabetic drug metformin suppresses self-renewal and proliferation of trastuzumab-resistant tumor-initiating breast cancer stem cells. Breast Cancer Research and Treatment 2011; 126: 355-364

[33] Cufí S, Corominas-Faja B, Vazquez-Martin A et al. Metformin-induced preferential killing of breast cancer initiating CD44 + CD24 - /low cells is sufficient to overcome primary resistance to trastuzumab in HER2 + human breast cancer xenografts. Oncotarget 2012; 3: 395

[34] Ma J, Guo Y, Chen S et al. Metformin enhances tamoxifen-mediated tumor growth inhibition in ER-positive breast carcinoma. BMC Cancer 2014; 14: 172

[35] Jang SY, Kim A, Kim JK et al. Metformin inhibits tumor cell migration via down-regulation of MMP9 in tamoxifen-resistant breast cancer cells. Anticancer Research 2014; 34: 4127-4134
[36] Rocha GZ, Dias MM, Ropelle ER et al. Metformin amplifies chemotherapy-induced AMPK activation and antitumoral growth. Clinical Cancer Research 2011; 17: 3993-4005

[37] Cantrell LA, Zhou C, Mendivil A et al. Metformin is a potent inhibitor of endometrial cancer cell proliferation-implications for a novel treatment strategy. Gynecologic Oncology 2010; 116: 92-98

[38] Kim S, Choi JH, Lim HI et al. Silibinin prevents TPA-induced MMP-9 expression and VEGF secretion by inactivation of the Raf/MEK/ERK pathway in MCF-7 human breast cancer cells. Phytomedicine 2009; 16: 573-580

[39] Lu W, Lin C, King TD et al. Silibinin inhibits Wnt/ $\beta$-catenin signaling by suppressing Wnt co-receptor LRP6 expression in human prostate and breast cancer cells. Cellular Signalling 2012; 24: 2291-2296 